\title{
Relation of Surface T-wave to Vulnerability to Ventricular Fibrillation in Explanted Structurally Normal Hearts
}

\author{
Marianna Meo ${ }^{1,2,3}$, Pietro Bonizzi ${ }^{4}$, Laura R Bear ${ }^{1,2,3}$, Matthijs Cluitmans ${ }^{5}$, Emma Abell ${ }^{1,2,3}$, Michel \\ Haiissaguerre $^{1,2,3,6}$, Olivier Bernus ${ }^{1,2,3}$, Rémi Dubois ${ }^{1,2,3}$ \\ ${ }^{1}$ IHU Liryc, Electrophysiology and Heart Modeling Institute, FBU, Pessac-Bordeaux, France \\ ${ }^{2}$ Univ Bordeaux, CRCTB, U1045, Bordeaux, France \\ ${ }^{3}$ INSERM, CRCTB, U1045, Bordeaux, France \\ ${ }^{4}$ Department of Data Science and Knowledge Engineering, Maastricht University, Maastricht, the \\ Netherlands \\ ${ }^{5}$ Department of Cardiology, Cardiovascular Research Institute Maastricht (CARIM), Maastricht \\ University Medical Center, Maastricht, the Netherlands \\ ${ }^{6}$ Bordeaux University Hospital (CHU), Electrophysiology and Ablation Unit, Pessac, France
}

\begin{abstract}
Introduction: Body surface characterization of repolarization gradient substrates for ventricular fibrillation $(V F)$ is still poor. We investigated if VF propensity can be assessed from surface T-wave in an ex-vivo model of purely electrical repolarization dispersion. Methods: To create repolarization gradients, dofetilide and/or pinacidil were separately infused in $N=7$ isolated pig hearts in a humanshaped torso tank. At each drug state, VF inducibility was quantified by the vulnerability window $(V W)$, i.e. the interval during which VF was triggered by $S 1$ (atrium) S2 (ventricle) stimulation. $T$-wave duration (the peak-to-end interval, $\mathrm{T}_{\mathrm{PEAK}}-\mathrm{T}_{\mathrm{END}}$ ), and shape, i.e., symmetry (the ratio of the areas under the peak-to-end and onset-to-peak frames, Asy), and flatness (kurtosis, Kurt) were computed from 256 tank potentials. We fitted a linear mixed-effect (LMM) model to link $T$-wave markers (fixed effects) with $V W$ (response), with random effects on drug states and hearts. Results: VF was induced in 14/23 drug states from at least one ventricle. In vulnerable substrates (higher $V W$ ), $T$ waves were longer $\left(\mathrm{T}_{\mathrm{PEAK}}-\mathrm{T}_{\mathrm{END}}, p=0.0004\right)$, less symmetric (Asy, $p<0.0001$ ) and moderately flat (Kurt, $p=0.1$ ). In a combined LMM model, significant effects were only explained by Asy $(p<0.0001)$ and Kurt $(p=0.04)$. Conclusions: Vulnerability to VF can be assessed from surface $T$-wave in heterogeneous repolarization substrates.
\end{abstract}

\section{Introduction}

Sudden cardiac death (SCD) is a major cause of mortality, accounting for 15-20\% of all deaths worldwide [1].
In SCD survivors, ventricular fibrillation (VF) is often the lethal heart rhythm disorder identified at the time of event [2], and in a considerable portion of these victims no overt structural heart diseases have been previously diagnosed [3]. Abnormal dispersion of ventricular repolarization provides a favorable substrate for the initiation of malignant arrhythmias, as a premature beat interacting with heterogeneous repolarization substrates can readily initiate re-entry [4]. Surface electrocardiogram analysis is a promising tool for the investigation of such alterations [5]. However, how repolarization disturbances reflect on the body surface and increase arrhythmogenesis in apparently normal hearts still needs to be elucidated. In this study we use an ex-vivo model of purely electrical repolarization heterogeneity to investigate whether surface T-wave analysis can help identifying vulnerable VF substrates.

\section{Methods}

\subsection{Experimental setup and dataset}

The experimental protocol was approved by Directive 2010/63/EU of the European Parliament on the protection of animals used for scientific purposes and the local ethical committee. As in [6,7], $N_{H}=7$ Langendorff-perfused pig hearts were suspended in a human-shaped torso tank filled with Tyrode's solution. Repolarization gradients were created by separate infusion of pinacidil $(30 \mu \mathrm{M}$, shortening repolarization duration) and/or dofetilide $(250 \mathrm{nM}$, prolonging repolarization duration) through the left anterior descending (LAD) and non-LAD coronaries, respectively. 


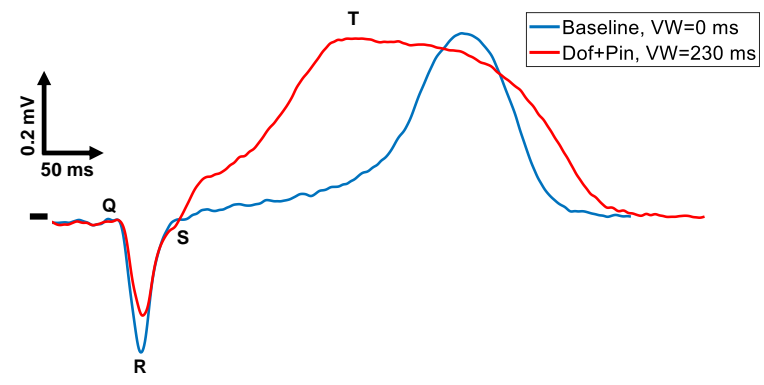

Figure 1. Two representative single-lead QRST sequences from a tank potential recorded from an experiment at baseline, without inducible VF (Baseline, VW=0 ms, blue trace), and after creating repolarization gradients by simultaneous perfusion with dofetilide and pinacidil, with high susceptibility to VF (Dof+Pin, VW=230 ms, red trace).

\subsection{VF induction testing}

At each drug state, VF inducibility was tested by programmed stimulation. A single S2 ventricular extrastimulus (mimicking a premature ventricular contraction) was delivered after a train of eight $\mathrm{S} 1$ drive beats at a cycle length of $600 \mathrm{~ms}$ in the right atrium. As in [8], if S2 could not trigger VF, the S1-S2 interval was progressively decremented by $10-\mathrm{ms}$ steps, until arrhythmia was induced or $\mathrm{S} 2$ did not result in capture. The protocol was repeated for different pacing locations, including the posterior wall outside the LAD (with longer repolarization during dofetilide perfusion), and the anterior left or right ventricular wall in the LAD area (with shorter repolarization during pinacidil perfusion). The degree of susceptibility to VF was quantified by the vulnerability window, i.e. the duration of the interval during which VF was triggered by S2 ventricular extra-beats at a specific experimental stage.

\subsection{Torso signal preprocessing and T-wave feature extraction}

Prior to VF induction, at each experimental step, tank potentials (BioSemi, the Netherlands) were recorded over $N_{b}=10$ beats in sinus rhythm from $L=256$ electrodes embedded in the surface at a sampling frequency of $2048 \mathrm{~Hz}$. Noise filtering and detection of QRST fiducial points from torso signals were performed as in [7]. Too noisy or lowvoltage signals were discarded after visual inspection.

Two representative single-lead beats with their fiducial points are displayed before and after drug injection from the same experiment in Figure 1, in conditions of noninducible and inducible VF, respectively. In [7], we demonstrated that increased heterogeneity of repolarization is responsible for severe alterations of T-wave duration and morphology. Moving from this research, the present study investigates whether these T-wave markers may bring additional insights into the propensity to develop trigger-induced arrhythmias in these abnormal substrates. Specifically, as in [7], we measured the duration of the peak-to-end interval of the T-wave signal $\mathbf{s}_{\ell, \mathbf{i}}$ from each heartbeat $i=1, \ldots, N_{b}$ and tank electrode $\ell,=1, \ldots, L$, and its median value over all electrodes was considered as a global metric of T-wave duration $\left(\mathrm{T}_{\mathrm{PEAK}}-\mathrm{T}_{\mathrm{END}}\right)$.

T-wave morphological aberrations have been associated with abnormal dispersion of electrical recovery [7,9]. As in [7], in each tank electrode $\ell=1, \ldots, L$ and heartbeat $i=1, \ldots, N_{b}$ we quantified T-wave asymmetry with respect to its peak as:

$$
A s y_{\ell, i}=\frac{\int_{T_{P E A K}}^{T_{E N D}} s_{\ell, i}(t) d t}{\int_{T_{O N}}^{T_{P E A K}} s_{\ell, i}(t) d t},
$$

with $T_{O N}, T_{P E A K}$ and $T_{E N D}$ denoting the onset, peak and offset of T-wave, respectively. Index values closer to unity render more symmetric T-waves and are associated with less arrhythmogenic substrates. The median of all $A s y_{\ell, i}$ value over tank electrodes was used as a marker of VF vulnerability and denoted Asy.

Since flattened T-waves have been observed in patients with abnormal repolarization patterns, such as in type- 2 long-QT syndrome [10,11], and amplitude metrics have been regarded as predictive of SCD in the general population [12], in each tank electrode $\ell=1, \ldots, L$ and beat $i=1, \ldots, N_{b}$ we assessed the degree of T-wave flatness using the kurtosis index:

$$
\operatorname{Kurt}_{\ell, i}=\frac{\int_{T_{O N}}^{T_{E N D}}\left(t-\bar{s}_{\ell, i}\right)^{4} s_{\ell, i}(t) d t}{\left[\int_{T_{O N}}^{T_{E N D}}\left(t-\bar{s}_{\ell, i}\right)^{2} s_{\ell, i}(t) d t\right]^{2}},
$$

with $\bar{s}_{\ell, i}$ equal to the temporal average of the T-wave at the $i$ th beat and the $l$ th electrode. Lower kurtosis values were associated with flattened and broader T-waves, which are typically considered arrhythmogenic. The spatial median of all T-wave kurtosis values at each heartbeat was used as an index of VF propensity (Kurt).

\subsection{Statistical assessment of VF inducibil- ity from surface $T$-wave}

To understand to which extent surface signal features can measure the propensity to develop VF in substrates with different degrees of repolarization dispersion, we fitted a linear mixed-effect (LMM) model describing the relationship between T-wave indices and VW markers of VF inducibility. A LMM model was preferred to a standard linear regression approach due to the hierarchical nature of our experimental dataset, with the following formulation:

$$
\mathbf{y}=\mathbf{X b}+\mathbf{Z}_{\mathbf{H}} \mathbf{u}_{\mathbf{H}}+\mathbf{Z}_{\mathbf{D S}} \mathbf{u}_{\mathbf{D S}}+\boldsymbol{\epsilon} .
$$


The $N_{y} \times 1$ response vector $\mathbf{y}$ represents the set of all VW measurements. T-wave markers were considered as LMM fixed effects, and given by the product between the $N_{y} \times N_{p}$ matrix $\mathbf{X}$ of the $N_{p}$ predictor variables, and the $N_{p} \times 1$ vector of the fixed-effects regression coefficients $\mathbf{b}$. Two types of random effects were included in our LMM model. First, to account for inter-experiment variability across the database, the first random effect was assumed to be due to the $N_{H}=7$ experimental hearts, and equal to the product between the $N_{y} \times N_{H}$ design matrix $\mathbf{Z}_{\mathbf{H}}$ and the $N_{H} \times 1$ vector of regression coefficients $\mathbf{u}_{\mathbf{H}}$. Secondly, to quantify different levels of repolarization heterogeneity due to the pharmacological interventions performed within the same experiment (intra-subject variability), drug states were also modeled as random effects and quantified by the product between the $N_{y} \times N_{D S}$ design matrix $\mathbf{Z}_{\mathbf{D S}}$ and the $N_{D S} \times 1$ vector of regression coefficients $\mathbf{u}_{\mathbf{D S}}$, with $N_{D S}=8$ equal to the total number of experimental stages based on drug type and doses. Finally, the term $\epsilon$ stands for the residual information of $\mathbf{y}$ that is not explained by the LMM model. LMM regression parameters were determined by maximum log-likelihhod estimation.

Each surface signal parameter was incorporated as a fixed effect into the LMM model separately $\left(N_{p}=1\right)$. A multivariate combination of T-wave indices was tested as well $\left(N_{p}=3\right)$. To assess the validity of each model, the significance of the fixed effect regression slopes against zero was tested. Moreover, a likelihood ratio (LR) test was performed to evaluate whether LMM goodness-of-fit may benefit from expressing VW as a combination of multiple T-wave parameters rather than considering each of them separately. For each comparison between uni- and multivariate LMM models, we computed the LR test statistic, i.e., the difference of their deviances, and the Bonferroniadjusted $p$-value for multiple comparisons [13]. All tests were statistically significant if $p<0.05$.

\section{Results}

VF was successfully induced in 14/23 drug states from at least one ventricle. More vulnerable substrates were associated with drug-induced repolarization gradients, and VF could be triggered from multiple sites, thus yielding longer VWs than in the absence of drugs ( $82 \pm 88 \mathrm{~ms}$ vs 7 $\pm 13 \mathrm{~ms}, p<0.02$ ). In 6 cases ( 3 hearts) VF was triggered only from one ventricle ( 3 from LV and 3 from RV, while the other one was refractory to stimulation) in the LAD area (short repolarization duration), with short VWs (between 10 and $40 \mathrm{~ms}$ ). VF could be initiated from both ventricles in 8 drug states from 4 hearts, yielding higher VW values (from 70 to $230 \mathrm{~ms}$ ). In the remaining cases (from 4 experiments), the hearts were resistant to VF induction, regardless of the pacing site. Of note, VF was never triggered by the extra-LAD ventricular regions (i.e. those perfused by dofetilide, with prolonged repolarization).

The outcome of the LMM fitting to VW experimental measures for each surface T-wave marker is displayed in Figure 2, with LMM marginal responses and 95\% confidence interval (CI) limits accounting for fixed effects only. When
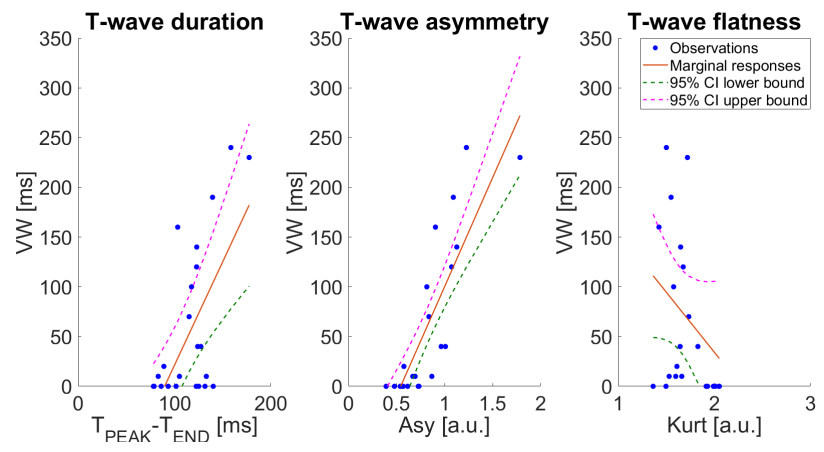

Figure 2. Marginal responses and 95\% CI bounds of the LMM models describing the relationship between VW experimental measures (blue dots) and surface repolarization markers: $T_{\text {PEAK}}-T_{\text {END }}$ (left panel), Asy (middle) and Kurt (right panel).

the hearts were more prone to develop VF (i.e. when VW was higher), a significant prolongation of the terminal portion of T-wave was observed on the body surface and quantified by higher $T_{\text {PEAK }}-T_{\text {END }}$ values $(p=0.0004)$. Increased susceptibility to VF was also associated with more pronounced T-wave morphological aberrations. Indeed, in more vulnerable substrates T-waves were more asymmetric, due to a steeper T-wave upslope and a more trapezoidal/triangular waveform shape, and assessed by remarkably higher Asy values $(p<0.0001)$. Furthermore, in inducible cases T-waves were less peaked, with an inverse linear relation between the VW and Kurt indices, although more moderate compared with the other signal parameters $(p=0.14)$. When all T-wave indices were simultaneously included into the same LMM model, significant effects were explained by T-wave shape, both in terms of symmetry (Asy, $p<0.0001$ ) and flatness (Kurt, $p=0.04$ ), but not duration $\left(\mathrm{T}_{\mathrm{PEAK}}-\mathrm{T}_{\mathrm{END}}, p=0.42\right.$ ). In addition, the outcome of the LR test in Table 1 confirms that combining multiple T-wave indices can improve the characterization of vulnerable substrates from the body surface. Prediction accuracy of the LMM models based on the $\mathrm{T}_{\mathrm{PEAK}}-\mathrm{T}_{\mathrm{END}}$ and the Kurt indices only is significantly improved by parameters' combination, whereas the Asy-based model seems to perform similarly to the multivariate one.

\section{Discussion and conclusions}

Despite recent advances in VF management, we still lack strategies for the assessment of the arrhythmic risk in patients with apparently normal hearts, for whom effec- 


\begin{tabular}{|c|c|c|}
\hline T-wave index & LR test statistic & p-value \\
\hline \hline $\mathrm{T}_{\mathrm{PEAK}}-\mathrm{T}_{\mathrm{END}}$ & 20.63 & $\mathbf{0 . 0 0 6}$ \\
\hline Asy & 4.49 & 0.24 \\
\hline Kurt & 28.63 & $\mathbf{0 . 0 0 3}$ \\
\hline
\end{tabular}

Table 1. LR test statistic and $p$-value for the comparison between uni- and multivariate LMM models; significant $p$ values are in boldface.

tive diagnostic tools are still missing [2]. Dispersion of ventricular repolarization often underlies these undetected abnormalities and predisposes to arrhythmias [14]. While several studies support the use of surface T-wave analysis as a potential noninvasive screening tool $[4,5,10]$, it is still unclear whether and how VF susceptibility can be assessed on the body surface and how VF trigger timing and location affect electrical signals. Using an experimental model of solely electrical repolarization dispersion, in line with [14], our findings confirm that VF initiation results from the combination of a sharp repolarization gradient with a timed premature trigger from an early-repolarizing ventricular region. These specific conditions are responsible for significant changes in surface T-wave, that are accurately captured by the proposed signal metrics and correlate with the VW marker of VF inducibility. We observed a marked prolongation of the $\mathrm{T}_{\mathrm{PEAK}}-\mathrm{T}_{\mathrm{END}}$ interval, a widely investigated marker of global unevenness of repolarization [14], in keeping with clinical studies on Brugada and long QT syndrome [15]. In our dataset, drug-induced repolarization dispersion was also responsible for the genesis of broad-based and asymmetric T-waves, which are also typical electrocardiographic manifestations of long QT syndrome (type 2) [10,11]. Alterations in T-wave duration and shape prove not only to be able to capture different degrees of repolarization heterogeneity [7], but also to distinguish between vulnerable and non-vulnerable substrates, with linear dependence of T-wave metrics on measures of tissue arrhythmogenesis. We provided experimental validation of surface T-wave analysis for VF susceptibility assessment, with potential application to clinical patients with structurally normal hearts in future works.

\section{Acknowledgements}

This study was supported by the French Government as part of the "Investments of the Future" program managed by the National Agency Research (ANR-10-IAHU04LIRYC), and the Leducq transatlantic network of excellence RHYTHM transatlantic network (16CVD02).

\section{References}

[1] Adabag AS, Luepker RV, Roger VL, et al. Sudden cardiac death: epidemiology and risk factors. Nature Reviews Car- diology 2010;7:216-225.

[2] Haïssaguerre M, Duchateau J, Dubois R, et al. Idiopathic ventricular fibrillation: Role of purkinje system and microstructural myocardialabnormalities. Journal of the American College of Cardiology Clinical Electrophysiology 2020;6(6):591-608.

[3] Modi S, Krahn AD. Sudden cardiac arrest without overt heart disease. Circulation 2011;123(25):2994-3008.

[4] Sheridan PJ, Marques JLB, Newman CMH, et al. Ratedependent measures of repolarization predict inducibility of ventricular arrhythmias. Europace 2010;12:553-560.

[5] Prenner SB, Shah SJ, Goldberger JJ, Sauer AJ. Repolarization heterogeneity: beyond the QT interval. Journal of the American Heart Association 2016;5(5):e003607.

[6] Bear LR, Bouhamama O, Cluitmans M, et al. Advantages and pitfalls of noninvasive electrocardiographic imaging. Journal of Electrocardiology 2019;57S:S15-S20.

[7] Meo M, Bonizzi P, Bear LR, et al. Body surface mapping of ventricular repolarization heterogeneity: an ex-vivo multiparameter study. Frontiers in Physiology 2020;11:933.

[8] Arevalo HJ, Vadakkumpadan F, Guallar E, et al. Arrhythmia risk stratification of patients after myocardial infarction using personalized heart models. Nature Communications 2016;7:11437.

[9] di Bernardo D, Murray A. Explaining the T-wave shape in the ECG. Nature 2000;403(103):40.

[10] Vicente J, Johannesen L, Mason JW, et al. Comprehensive $\mathrm{T}$ wave morphology assessment in a randomized clinical study of dofetilide, quinidine, ranolazine, and verapamil. Journal of the American Heart Association 2015; 4(4):e001615.

[11] Porta-Sánchez A, Spillane DR, Harris L, et al. T-wave morphology analysis in congenital long QT syndrome discriminates patients from healthy individuals. JACC Clinical Electrophysiology 2017;3(4):374-381.

[12] Kenttä TV, Sinner MF, Nearing BD, et al. Repolarization heterogeneity measured with t-wave area dispersion in standard 12-lead ecg predicts sudden cardiac death in general population. Circulation Arrhythmia and Electrophysiology 2018;11(2):e005762.

[13] Khuri AI, Mathew T, Sinha BK. Statistical tests for mixed linear models, volume 906. John Wiley \& Sons, 2011.

[14] Coronel R, Wilms-Schopman FJG, Opthof T, Janse MJ. Dispersion of repolarization and arrhythmogenesis. Heart Rhythm 2009;6:537-543.

[15] Tse G, Yan BP. Traditional and novel electrocardiographic conduction and repolarization markers of sudden cardiac death. EP Europace 2017;19(5):712-721.

Address for correspondence:

Marianna Meo

IHU LIRYC Av. Hâut Lèvêque 33604 Pessac-Bordeaux, France marianna.meo@ihu-liryc.fr 